\title{
Enhancement of small doppler frequencies detection for LFMCW radar
}

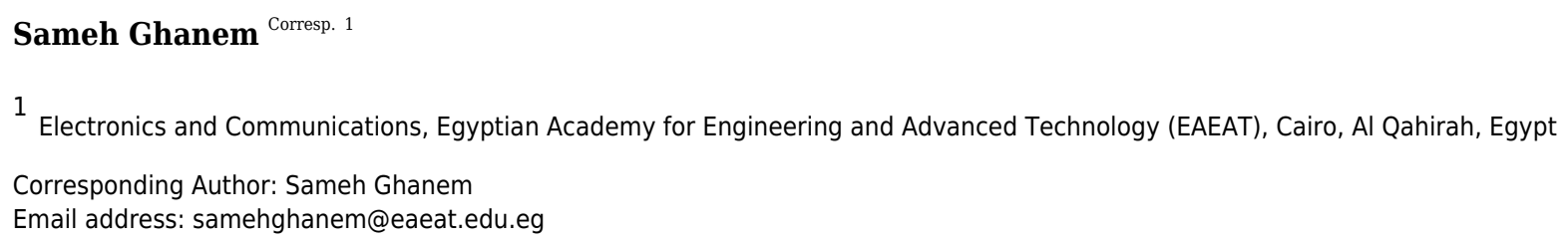

Open Access proceedings Journal of Physics: Conference series Detection of targets with small Doppler frequencies of Linear Frequency Modulated Continuous Wave (LFMCW) Radars is the main task of this paper. Moving Target Indicator (MTI) is used to reject the fixed targets and high-speed targets through the radar research area. In this work, targets with small Doppler frequencies can be detected perfectly based on the frequency response of Single Delay Line Canceller (SDLC) followed by Single Delay Line Integrator (SDLI). An enhancement of the proposed algorithm is achieved using a filter in the range direction of the range-Doppler processor scheme. The proposed filter is chosen with certain coefficients after the first Fast Fourier Transform (FFT) processor in range to enhance the radar performance. The evaluation of the proposed algorithm is achieved at different slow Doppler scenarios of the target and compared with the traditional algorithm which uses only MTI processor. Another aspect that is important for evaluation of the proposed algorithm is the detection performance of the algorithms through the Receiver Operating Characteristic (ROC) curves. Implementation of the proposed algorithm using FPGA is performed in real time applications and it is found that it meets the simulation results. 


\section{Enhancement of Small Doppler Frequencies Detection for LFMCW Radar}

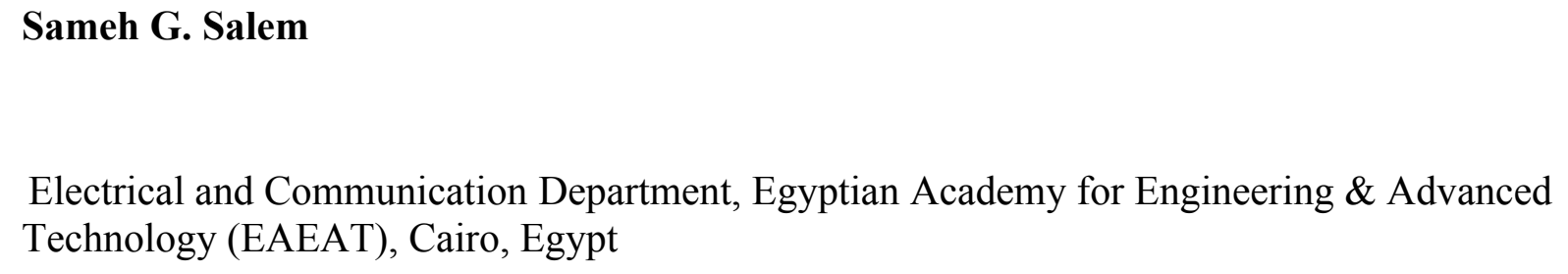

Electrical and Communication Department, Egyptian Academy for Engineering \& Advanced Technology (EAEAT), Cairo, Egypt

\section{Corresponding Author:}

\section{Sameh G. Salem}

Electrical and Communication Department, Egyptian Academy for Engineering \& Advanced Technology (EAEAT), Cairo, Egypt.

Email address: Samehghanem@,eaeat.edu.eg,Samehghanim1@gmail.com 


\title{
Enhancement of Small Doppler Frequencies Detection for LFMCW Radar
}

\author{
Author: Sameh G. Salem \\ 1,2 Egyptian Academy for Engineering \& Advanced Technology (EAEAT), Cairo, Egypt \\ Samehghanem@eaeat.edu.eg, Samehghanim1@,gmail.com
}

Abstract - Detection of targets with small Doppler frequencies of Linear Frequency Modulated Continuous Wave (LFMCW) Radars is the main task of this paper. Moving Target Indicator (MTI) is used to reject the fixed targets and high-speed targets through the radar research area. In this work, targets with small Doppler frequencies can be detected perfectly based on the frequency response of Single Delay Line Canceller (SDLC) followed by Single Delay Line Integrator (SDLI). An enhancement of the proposed algorithm is achieved using a filter in the range direction of the range-Doppler processor scheme. The proposed filter is chosen with certain coefficients after the first Fast Fourier Transform (FFT) processor in range to enhance the radar performance. The evaluation of the proposed algorithm is achieved at different slow Doppler scenarios of the target and compared with the traditional algorithm which uses only MTI processor. Another aspect that is important for evaluation of the proposed algorithm is the detection performance of the algorithms through the Receiver Operating Characteristic (ROC) curves. Implementation of the proposed algorithm using FPGA is performed in real time applications and it is found that it meets the simulation results.

Index Terms-LFMCW Radar, SDLC-MTI, Doppler Frequency, 2D-FFT, Signal processing.

\section{INTRODUCTION}

Detection of slow moving targets is an important for LFMCW radars based on traditional techniques such as Fast Fourier Transform (FFT) in both range and Doppler directions [1]. Usage of FMCW radar due to many advantages such as its small weight, small energy consumption, and less hardware complexity relative to other radars [2]. The target information such as range and speed can be extracted from LFMCW radars using two-dimensional FFT algorithm. MTI is used to distinguish between the fixed and moving targets. There are many researches that enhance the detection of LFMCW radars using different techniques. In [3], target detection of LFMCW radars is enhanced using Compressive Sensing theory in Doppler direction. In [4], the authors investigate the real time implementation of the proposed algorithm for LFMCW radar. An enhancement of target detection in both range and Doppler directions based on CS is shown in [5]. In [6], the author enhances the detection of slow Doppler frequencies based on frequency response of both SDLC and integrator. The authors in [7], achievement of Range-Doppler detection of automotive FMCW 
radar is performed to extract the target information based on FFT calculations.

In this paper, an enhancement of small Doppler target detection is achieved using a proposed filter in range direction of FFT processor. The evaluation of the proposed processor has performed using MATLAB simulation and ROC curves. Implementation of the proposed processor is designed and tested using FPGA. The organization of this paper is achieved as follows; after the introduction, section 2 introduces a review on LFMCW radar processing and detection. Section 3 illustrates on the operation of the proposed processor. Experimental results using MATLAB is illustrated in section 4 . Section 5 presents the hardware implementation of the proposed processor using FPGA. Finally, the conclusion comes in section 6 .

\section{LFMCW RADAR DETECTION AND PROCESSING}

The general block diagram of LFMCW radar is shown as in Fig. (1). It consists of a transmitter, a receiver, mixer, and Analog-to-Digital converter (A/D). The received radar signal is processed after digitization using $\mathrm{A} / \mathrm{D}$ converter in the form of base band signal. The target decision is made using Constant False Alarm Rate (CFAR) algorithm after Range-Doppler processing based on FFT.

The transmitted signal of an FMCW radar can be modulated as follow [9]:

$$
S_{T}(t)=A_{T} \cos \left(2 \pi f_{c} t+2 \pi \int_{0}^{t} f_{T}(\tau) d \tau\right)
$$

Where $f_{T}(\tau)=\frac{B}{T} \tau$ is the linear transmitted frequency as function of time, $f_{c}$ is the carrier frequency, $B$ is the bandwidth, $A_{T}$ is the transmitted signal amplitude, and $T$ is the time duration. The received signal after reflection with delay of $t_{d}=2 \cdot \frac{R_{o}+v t}{C}$ and Doppler shift of $f_{D}=-2 \cdot \frac{f_{c} v}{C}$, the received frequency can be expressed as:

$$
f_{R}(t)=\frac{B}{T}\left(t-t_{d}\right)+f_{D}
$$

Where $R_{o}$ is the initial target range and $v$ is the target velocity.

The received radar signal can be expressed as:

$$
\begin{aligned}
& S_{R}(t)=A_{R} \cos \left(2 \pi f_{c}\left(t-t_{d}\right)+2 \pi \int_{0}^{t} f_{R}(\tau) d \tau\right) \\
& =A_{R} \cos \left(2 \pi f_{c}\left(t-t_{d}\right)+\frac{B}{T}\left(\frac{1}{2} t^{2}-t_{d} \cdot t\right)+f_{D} \cdot t\right)
\end{aligned}
$$

Where $A_{R}$ represents the received signal amplitude. The target information can be obtained by mixing the transmitted and received signals in time domain and filtered using low-pass filter (LPF) to generate the intermediate frequency $(\mathrm{IF})$ signal $\mathrm{S}_{\mathrm{IF}}(\mathrm{t})$ as:

$$
S_{I F}(t)=\frac{1}{2} \cos \left(2 \pi\left(f_{c} \cdot \frac{2 R_{o}}{C}\right)+2 \pi\left( \pm \frac{2 R_{o}}{C} \cdot \frac{B}{T}+\frac{2 f_{c} v}{C}\right) t\right)
$$


129

130

131

132

133

134

135

136

137

138

139

140

141

142

143

144

145

146

147

148

149

150

151

152

153

154

155

156

157

158

159

160

161

162

163

164

165

166

167

168

169

170

171

172

173

The sign \pm represents up and down ramp respectively. Therefore, beat frequency $\left(f_{b}\right)$ can be obtained in the spectrum of the baseband signal as:

$$
f_{b}= \pm \frac{2 R_{o}}{C} \cdot \frac{B}{T}+\frac{2 f_{c} v}{C}
$$

The relation between the beat frequency $\left(f_{b}\right)$ and range $(R)$ for fixed target is given by $[8,9]$

$$
f_{b}=\frac{2 R f_{m} \Delta F}{C}
$$

Where $\mathrm{f}_{\mathrm{m}}$ is the modulated frequency, $\Delta \mathrm{f}$ is the receiver bandwidth and $\mathrm{C}$ is speed of light. Extraction of target information such as range and speed based on 2D-FFT is illustrated as shown in Fig. (2).

According to the traditional algorithm for LFMCW radar, the spectrum of received radar signal is processed using FFT in range direction followed by FFT in Doppler direction. The output of second FFT is applied to CFAR processor to make a decision for target detection. One of enhancement method for target detection using SDLC-MTI followed by integrator [6] is illustrated in Fig. (3).

The frequency response of SDLC MTI is multiplied with that of Single Delay Line Integrator (SDLI) as shown in Fig.(4). Fig.(5-a) represents the realization of stable SDLI and Fig.(5-b) illustrates its frequency response at different values of gain (A).

This structure has a good performance for slowly targets with small Doppler frequencies but has a bad evaluation for middle Doppler targets. This problem has been enhanced in [6] but with combined structure of the traditional algorithm (MTI with 2D-FFT processor) and the SDLI with Doppler FFT as shown in Fig.(6). The problem of this combination is the complexity which uses extra Doppler FFT processor in addition to SDLI processor. This problem can be overcame using the proposed processor or filter instead of high complexity as discussed in the next section.

\section{III.THE PROPOSED PROCESSOR}

Due to shortage of SDLC/SDLI algorithm in middle Doppler targets and expected high complexity in combination structure, the proposed processor is used to overcome this problem beside enhancement of off-pin targets as shown in Fig.(7).

The integrator of SDLC/SDLI has a stabilization factor, A, of one to ensure the system stability and the proposed filter is used as window function which multiply the incoming signal in time domain with the window function under consideration of same lengths. This multiplication in time domain can be obtained using convolution in frequency domain as in this case which spectral signal is more interest due to using FFT. The coefficients of the proposed filter is chosen to be 1 and -0.5 to solve the problem of middle Doppler frequencies. The proposed filter is chosen a head of first FFT processor which acts as a window function to ensure high detection capability before range-Doppler processor.

The realization of this filter is illustrated as in Fig.(8). For the proposed filter, the difference equation can be written as: 
174

175

176

177

178

179

180

181

182

183

184

185

186

187

188

189

190

191

192

193

194

195

196

197

198

199

200

201

202

203

204

205

206

207

208

209

210

211

212

213

214

215

216

217

218

219

$$
y(n)=x(n)-0.5 x(n-1)
$$

Where $x(n)$ and $y(n)$ represent the output of FFT processor and the output of the proposed filter respectively. The transfer function of the proposed filter can be written as:

$$
\begin{aligned}
& \text { Therefore, } \quad Y(Z)=X(Z)\left(1-0.5 Z^{-1}\right) \\
& H(Z)=1-0.5 Z^{-1}
\end{aligned}
$$

The proposed filter is chosen to enhance the detection capability of middle Doppler target velocities which improved using maximization process in [6] with approximately high complexity compared with that of the proposed filter. The simulation of the proposed processor performance and both SDLC/SDLI processor and the traditional algorithm based on MTI only is achieved and discussed in the next section.

Performance of the proposed processor is evaluated using simulation based on Matlab program. The performance is compared with that of both the traditional one and SDLC/SDLI algorithm under the same conditions. It is assumed that, the generation waveform is sawtooth with the central frequency of LFMCW radar (fc) is $24 \mathrm{GHz}$, bandwidth (B) is $20 \mathrm{MHz}$, modulation period (Tm) is $80 \mu \mathrm{sec}$, number of range cells is 1024 cells and number of Doppler cells is 32 cells. Comparison between the proposed processor and the traditional one which uses 2D-FFT processor is achieved as shown in Fig. (9). To study the effect of the proposed filter, two scenarios could be applied. First one, for off-pin targets and the other for middle-pin targets. The simulation is performed for these cases under the same conditions to verify a fair comparison.

\section{A. Off-pin targets}

The proposed filter has a great performance on the off-pin target detection. Assume a target in Doppler velocity equals $(4.5 / 15) \mathrm{f}_{\mathrm{m}}$ which is off-pin target which lies between Doppler velocities $(4 / 15) \mathrm{f}_{\mathrm{m}}$ and $(5 / 15) \mathrm{f}_{\mathrm{m}}$. The target can appear as two targets as in Fig. (10-a) using the traditional algorithm. But after applying the proposed filter, the target is located at one pin only (at pin number 5) or with Doppler velocity equals $(5 / 15) \mathrm{f}_{\mathrm{m}}$ as in Fig. (10-b) which indicates that, the proposed filter can resolve the problem of off-pin targets and therefore enhance the signal detection.

\section{B. Middle-pin targets}

To evaluate the effect of the proposed filter on the traditional algorithm, a set of moving targets are presented at different Doppler frequencies in noiseless environment $(1 / 32,4 / 32,8 / 32,12 / 32$, $16 / 32,20 / 32,24 / 32,28 / 32,31 / 32) * \mathrm{f}_{\mathrm{m}}$. Fig.(11) illustrates SDLC/SDLI processor response compared with the traditional algorithm at different Doppler frequencies. It is found that, there are no enhancement in target detection especially for middle-pin targets. Fig.(12) represents the response of the proposed algorithm based on the designed filter processor compared with the traditional one at different Doppler frequencies. It is clear that, the proposed processor based on 
220 filtering of the signal spectrum has a good performance for both off-pin targets and middle-pin maximization selection.

Another aspect to evaluate the proposed processor is the detection performance using ROC curve at different Doppler frequencies as shown in Fig. (13) and Fig.(14).

It is clear that, from Fig.(13), the detection performance of the proposed processor is enhanced compared with both the traditional and SDLC/SDLI processor by nearly $12 \mathrm{~dB}$ of SLC/SDLI processor and about $32 \mathrm{~dB}$ of the traditional algorithm at slow Doppler target velocity of $(2 / 32) \mathrm{f}_{\mathrm{m}}$. Fig.(14) illustrates that, the detection of the target enhanced using the proposed processor by nearly $38 \mathrm{~dB}$ of SLC/SDLI processor and about $10 \mathrm{~dB}$ of the traditional algorithm at middle Doppler target velocity of $(12 / 32) \mathrm{f}_{\mathrm{m}}$.

\section{HARDWARE IMPLMENTATION}

The implementation of the proposed processor is very important using FPGA which indicates that it can operate in real-time applications. The implementation is designed for the processing stage which includes; dechirping process of swatooth signal, 2D-FFT processor, proposed filter, MTI, SDLC/SDLI and CFAR detection. Xilinx KC705 DSP kit is used for implementation which includes KINTEX7 XC7K325T FPGA chip which has 241,152 logic cell, 768 DSP slices and about 216 Kbit RAM [10]. FPGA board is equipped with an FMC daughter board that contains TI's ADS62P49/ADS4249 dual-channel 14-bit 250Msps ADC and TI'sDAC3283 dual channel 16-bit $800 \mathrm{Msps}$ DAC on a daughter board [11]. The FFT core parameters are chosen to be; 32 number of samples, input data width is 32 bits, phase factor width is 24 bits, and Pipelined Streaming, I/O is used. The hardware implementation is performed for both the proposed processor and traditional algorithm which based on SDLC/SDLI. Two targets are simulated at Doppler velocity of pin (2/32) and the other target located at Doppler frequency pin number (12/32) as shown in Fig.(15) and Fig.(16). From these figures, it is clear that, the output of the proposed processor can improve the slowly moving target without any effect of other targets. The hardware specifications using Xilinx KC705 DSP kit is summarized in Table (1).

The verification of the implementation is performed using Chip scope for the two processors as shown in Fig.(17). It is found that, the Chip scope results met the simulation results as discussed before.

In this paper, detection of targets with small Doppler frequencies has been enhanced using a proposed processor. The enhancement has performed based on filtering process focusing on the detection based on the traditional algorithm using 2D-FFT processor and SDLC/SDLI processor. There are two main problems for target detection with small Doppler frequencies; first one, is the off-pin target detection which traditional algorithm cannot distinguish between these targets. The proposed processor can resolve this problem. Second problem, is the detection of middle-pin targets which is the main problem for SDLC/SDLI processor and this case has been overcame using maximization process but it suffer from high complexity. So, this problem can be resolved 
using the proposed algorithm based on a proposed filter as a head of the first FFT processor with less complexity compared with maximization process.

The performance of the proposed processor is examined compared with that of the traditional one and SDLC/SDLI processor through these two points. The detection performance of these targets can be evaluated using ROC curves at different target velocities and at low probability of false alarm.

It is found that, the detection performance of the proposed processor is enhanced by nearly $12 \mathrm{~dB}$ of SLC/SDLI processor and about nearly $32 \mathrm{~dB}$ of the traditional algorithm at slow Doppler target velocity and about nearly $38 \mathrm{~dB}$ of SLC/SDLI processor and $10 \mathrm{~dB}$ of the traditional algorithm at middle-Doppler target velocity. The implementation of the proposed processor is achieved using FPGA and Chip scope. It is found that, it meets the simulation results.

\section{REFERENCES}

[1] Merrill I. Skolnik, "Introduction to Radar systems", $3^{\text {rd }}$ edition, by The McGraw-Hill Companies, 2008.

[2] M. S. Lee and Y. H. Kim. "Design and Performance of a 24-GHz Switch-Antenna Array FMCW Radar System for Automotive Applications", IEEE Trans. on Vehicular Technology, Vol.59, pp.2290-2297, 2010.

[3] Sameh G. Salem, F. M. Ahmed, M. H. Ibrahim, A. H. Elbardawiny, "A Proposed Compressive Sensing Based LFMCW Radar Signal Processor", International Journal of Engineering Research \& Technology (IJERT), Vol. 4, Issue 04, P.611- 616, April-2015.

[4] Sameh G. Salem, Fathy M. Ahmed, Mamdouh H., Abdel Rahman H. Elbardawiny, "Design and Implementation of a New Approach of LFMCW Radar Signal Processing Based on Compressive Sensing in Azimuth Direction", IEEE International Radar Conference, Philadelphia, USA, pp.1-6, , May, 2016.

[5] M. H. Hossiny, S. G. Salem, F. M. Ahmed and K. H. Moustafa,"Enhance LFMCW radar detection and complexity using adaptive recovery CAMP algorithm", First International Workshop on Deep and Representation Learning (IWDRL), IEEE Publisher, pp. 1-6, Cairo 2018.

[6] F. M. Ahmed, "Detection of Targets with Small Apparent Doppler Frequencies in LFMCW Radars," \{IOP\} Conf. Ser. Mater. Sci. Eng., vol. 610, p. 12026, Oct. 2019.

[7] Winkler V,"Range Doppler detection for automotive FMCW radars". European Radar Conference (EuRAD), 10-12 Oct, 2007.

[8] Igor V.Komarov, Sergey M. Smolskiy, "Fundamental of short range FM radar", Published by Artech House ,2003.

[9] Nadav Levanon and Eli Mozeson, "Radar signals", Published by John Wiley \& Sons, Inc., Hoboken, New Jersey,2004.

[10] D. Challenges and X. Solutions, "KINTEX-7 FPGA KC705 Evaluation Kit: Versatile, HighPerformance Base Platform Shortens Time To Market For 7 Series Designs." Xilinx, Inc., 2012. 
306 [11] Abaco Systems," FMC150 for Digital Signal Processing”, User Manual, Abaco Systems, 307 USA, 2013. 


\section{Table $\mathbf{1}$ (on next page)}

FPGA utilization resources of the proposed processor. 
1

2

3
Table 1 FPGA utilization resources of the proposed processor

\begin{tabular}{|c|c|c|c|}
\hline Hardware Resources & Available resources & used & Utilization \\
\hline Slice Registers & 326,080 & 20697 & $5 \%$ \\
\hline Slice LUTs & 203800 & 43723 & $21 \%$ \\
\hline RAMB36E1/FIFO36E1s & 445 & 145 & $32 \%$ \\
\hline RAMB18E1/FIFO18E1s & 890 & 33 & $3 \%$ \\
\hline DSP48E1s & 840 & 345 & $40 \%$ \\
\hline
\end{tabular}


Figure 1

General_Block_Diagram_of_LFMCW_radar.

General_Block_Diagram_of_LFMCW_radar.

Transmititing

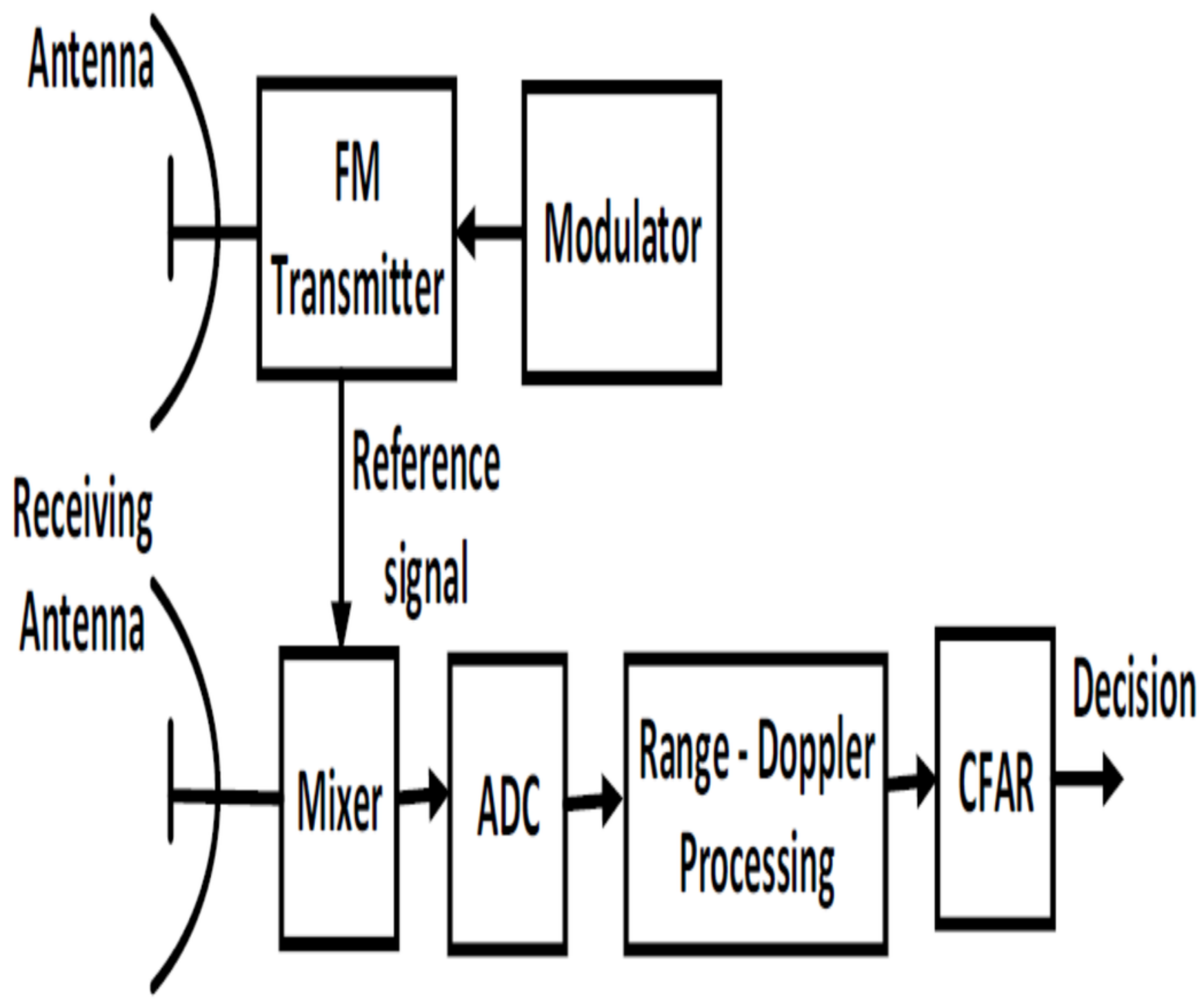


Figure 2

LFMCW_radar_signal_processing_using_2D FFT

LFMCW_radar_signal_processing_using_2D FFT

\section{frequency}
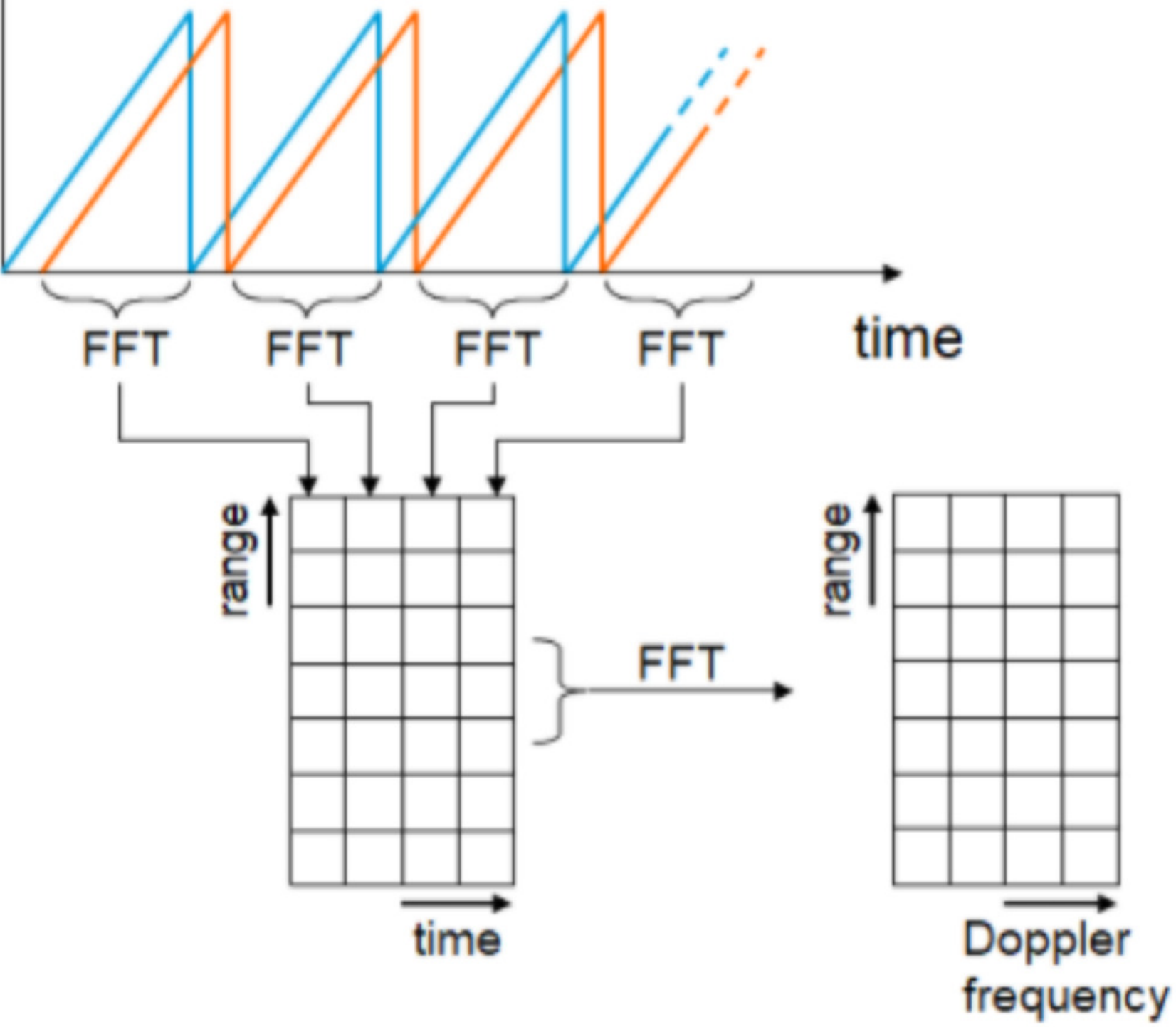
Figure 3

Block_diagram_of_SDLC

Block diagram of SDLC/SDLI algorithm

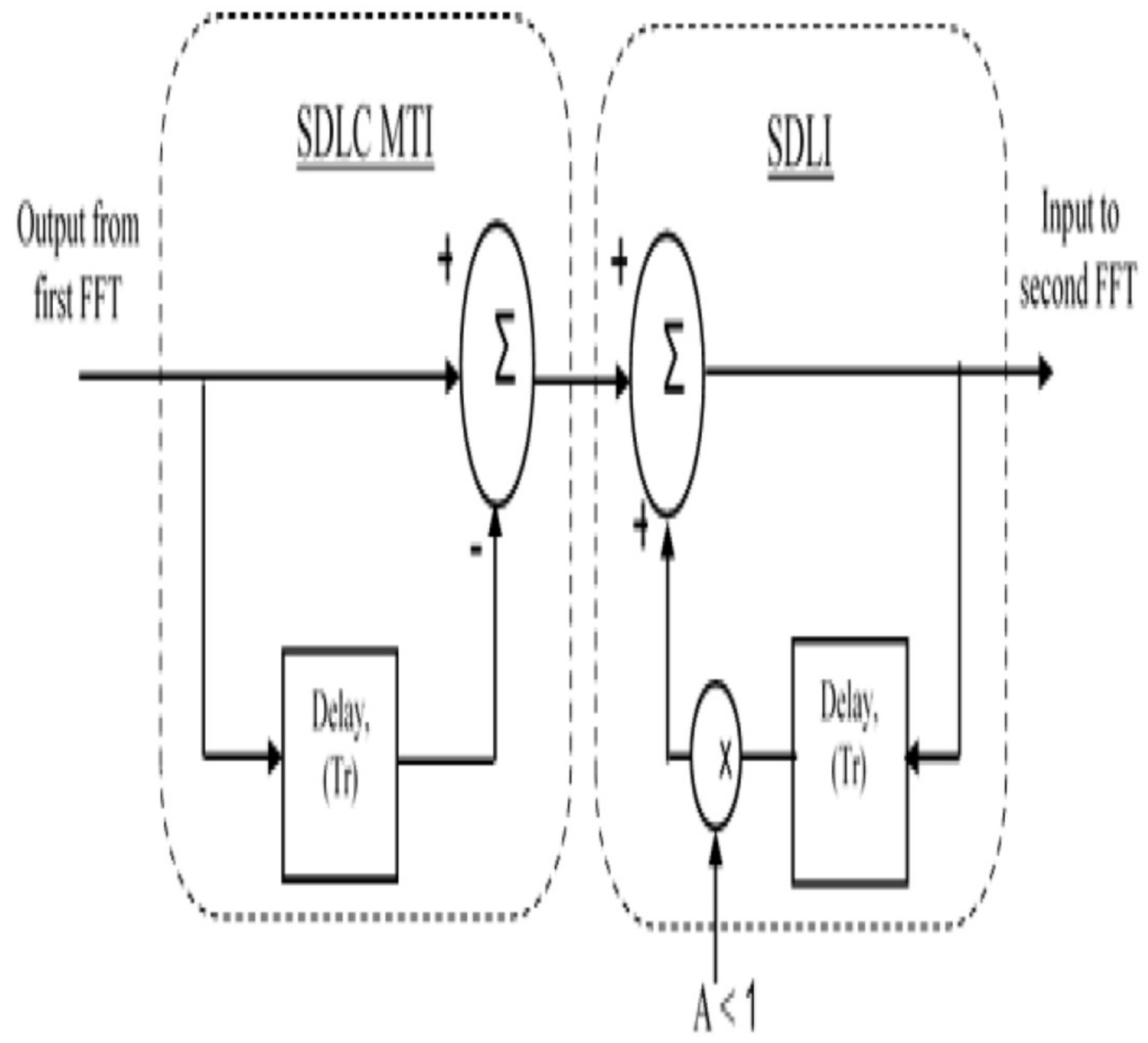


Figure 4

\section{Single_Delay_Line_Integrator_Structure}

Single_Delay_Line_Integrator_(SDLI)_structure:

(a)Stable Realization.

(b) Frequency response at different values of $\mathrm{A}$

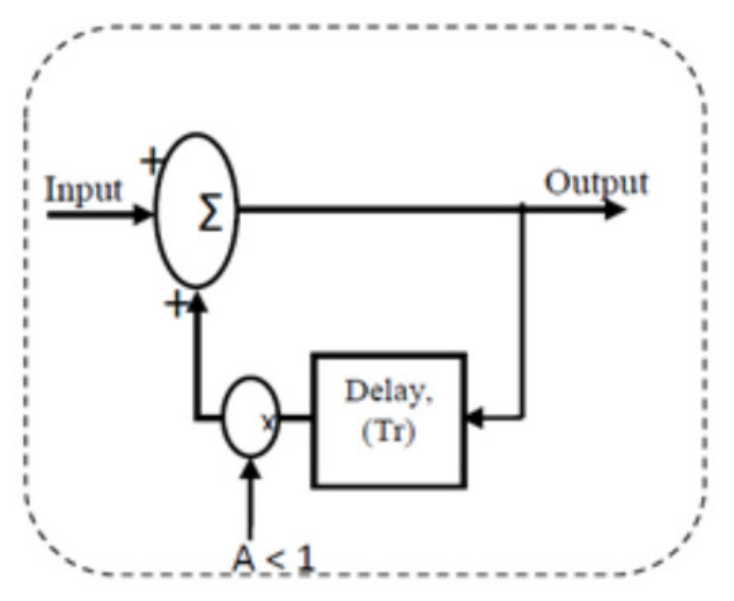

(a)

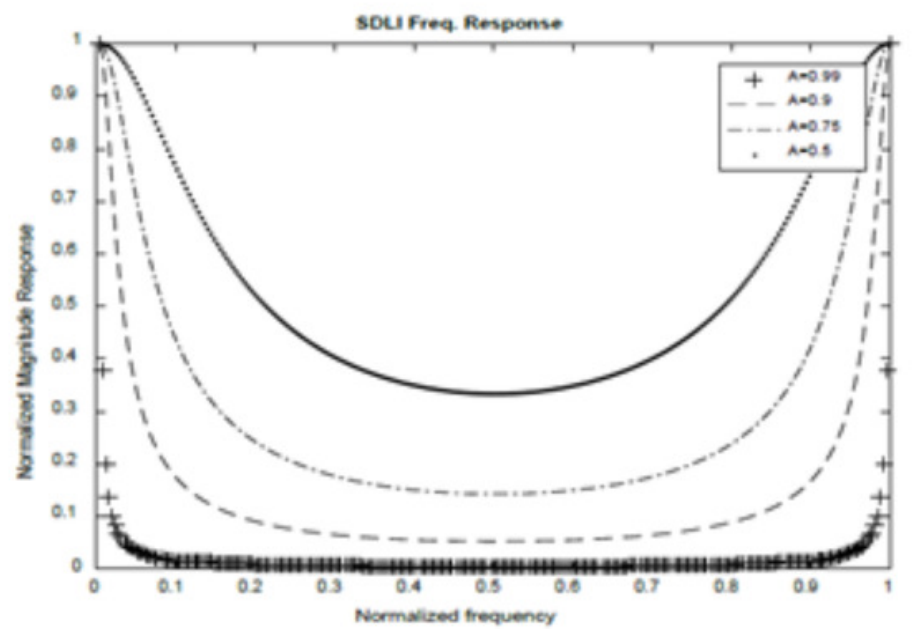

(b) 
Figure 5

LFMCW_radar_processor_based_on_SDLC

LFMCW radar processor based on SDLC/SDLI Processor

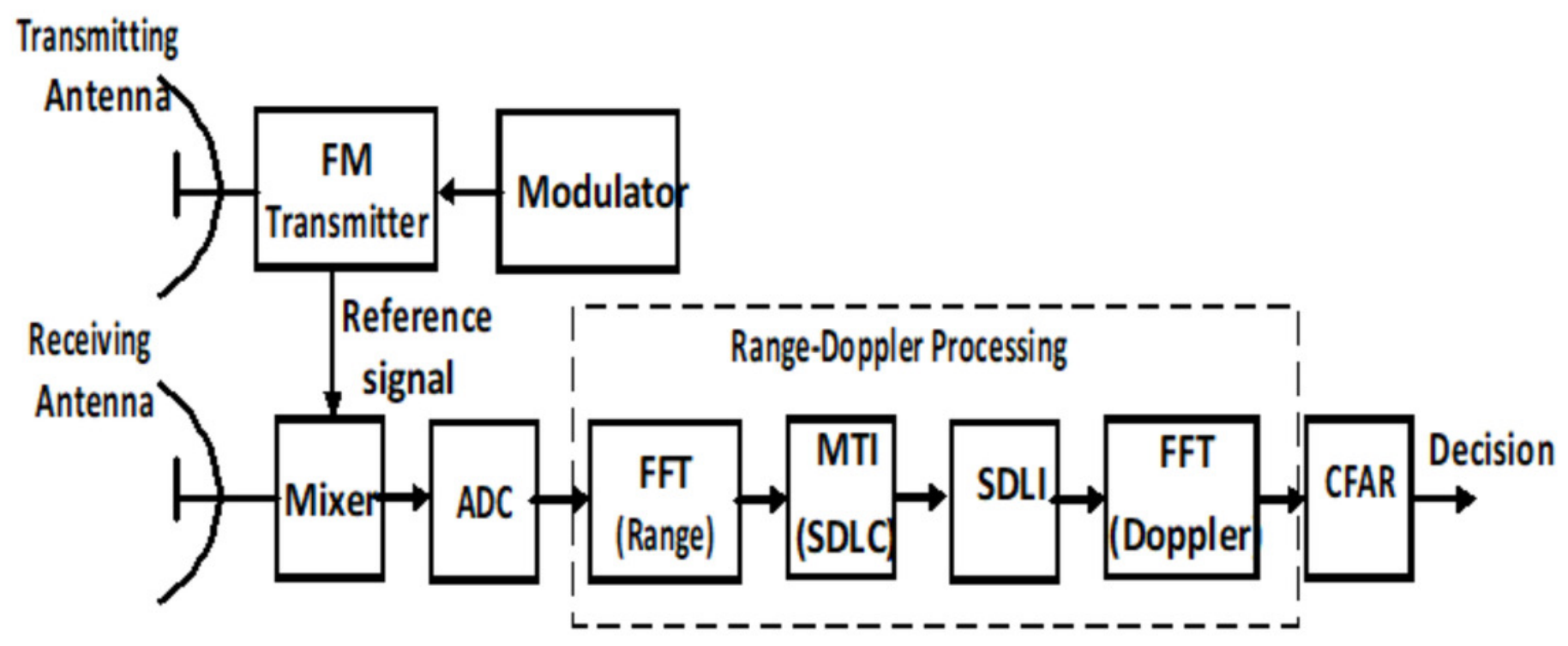


Figure 6

Block_diagram_of_LFMCW_radar_with_the_combined_structure

Block diagram of LFMCW radar with the combined structure

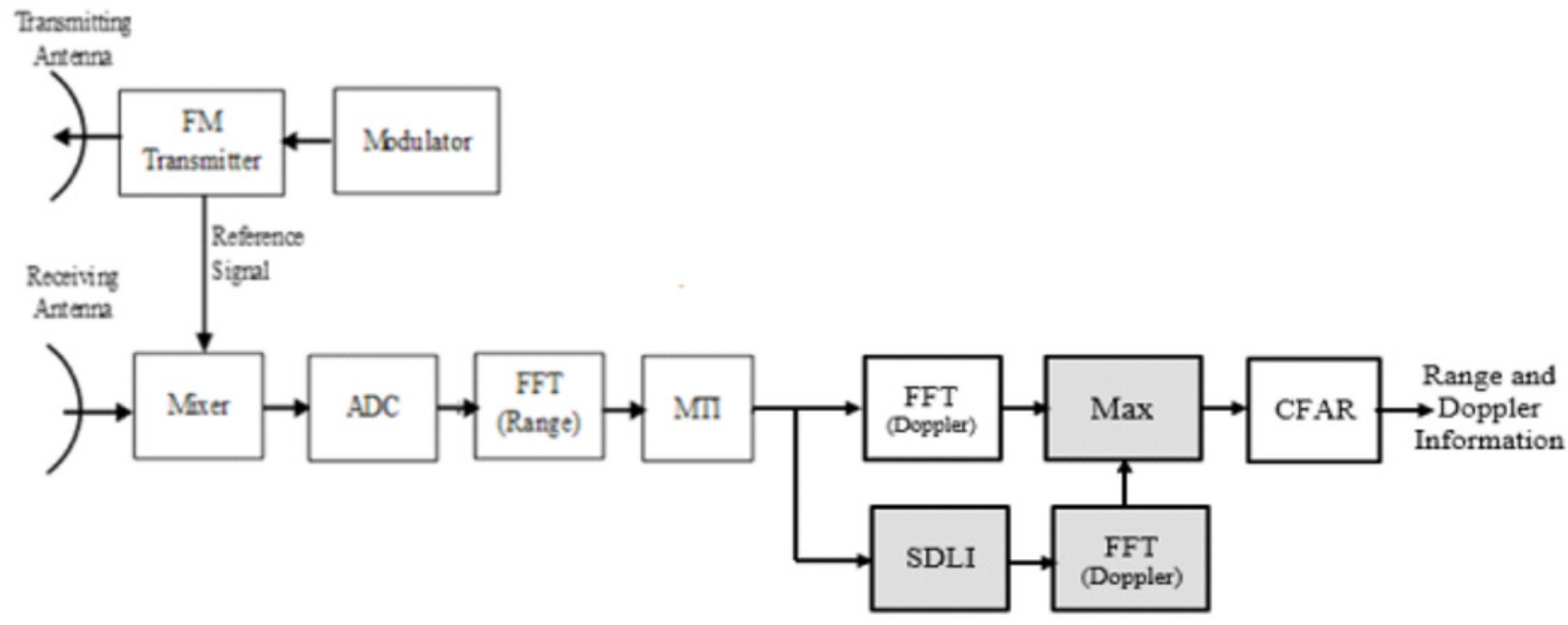


Figure 7

General_block_diagram_of_LFMCW_radar_using_the_proposed_processor

General block diagram of LFMCW radar using the proposed processor

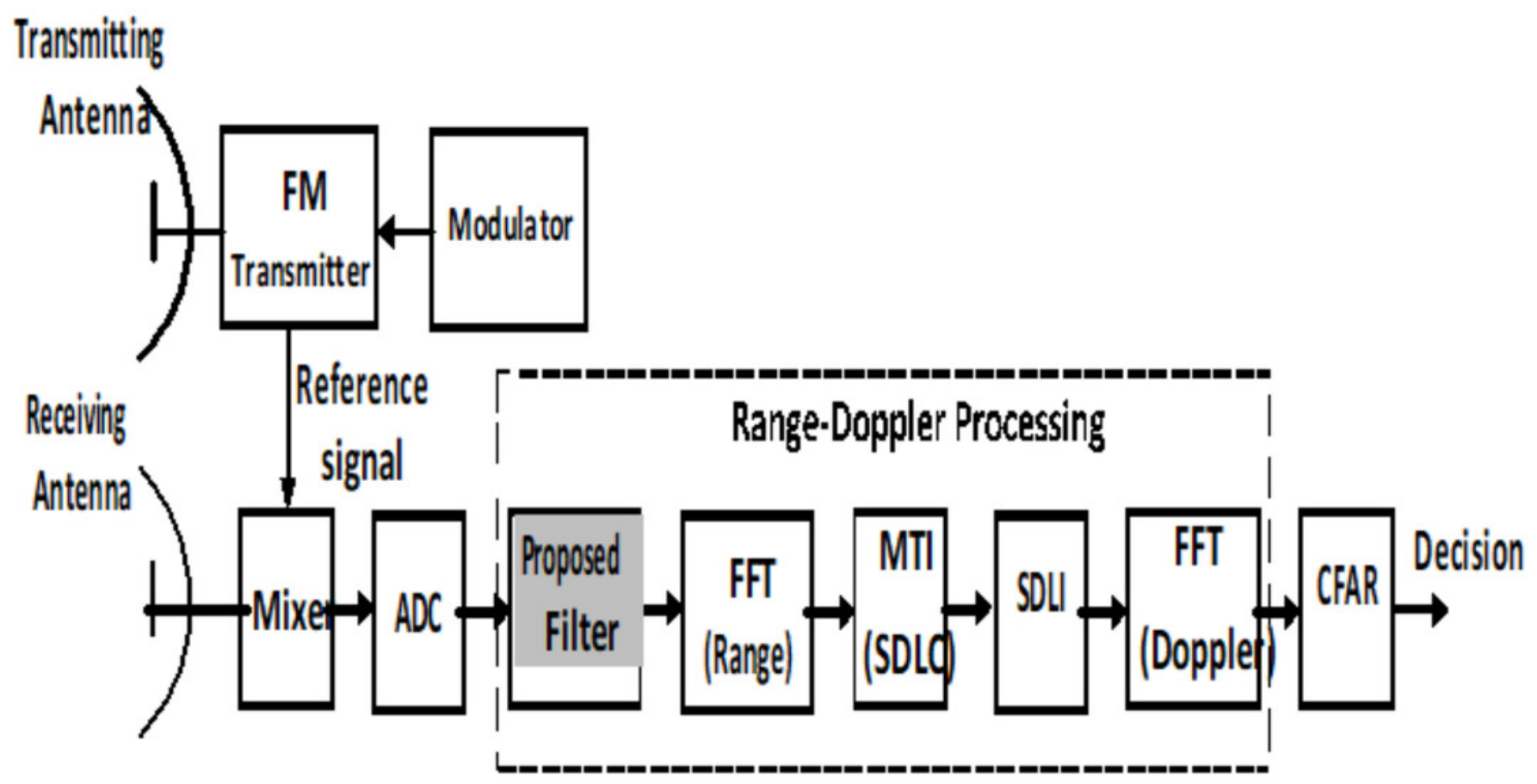


Figure 8

Realization_of_the_proposed_filter_processor

Realization of the proposed filter processor

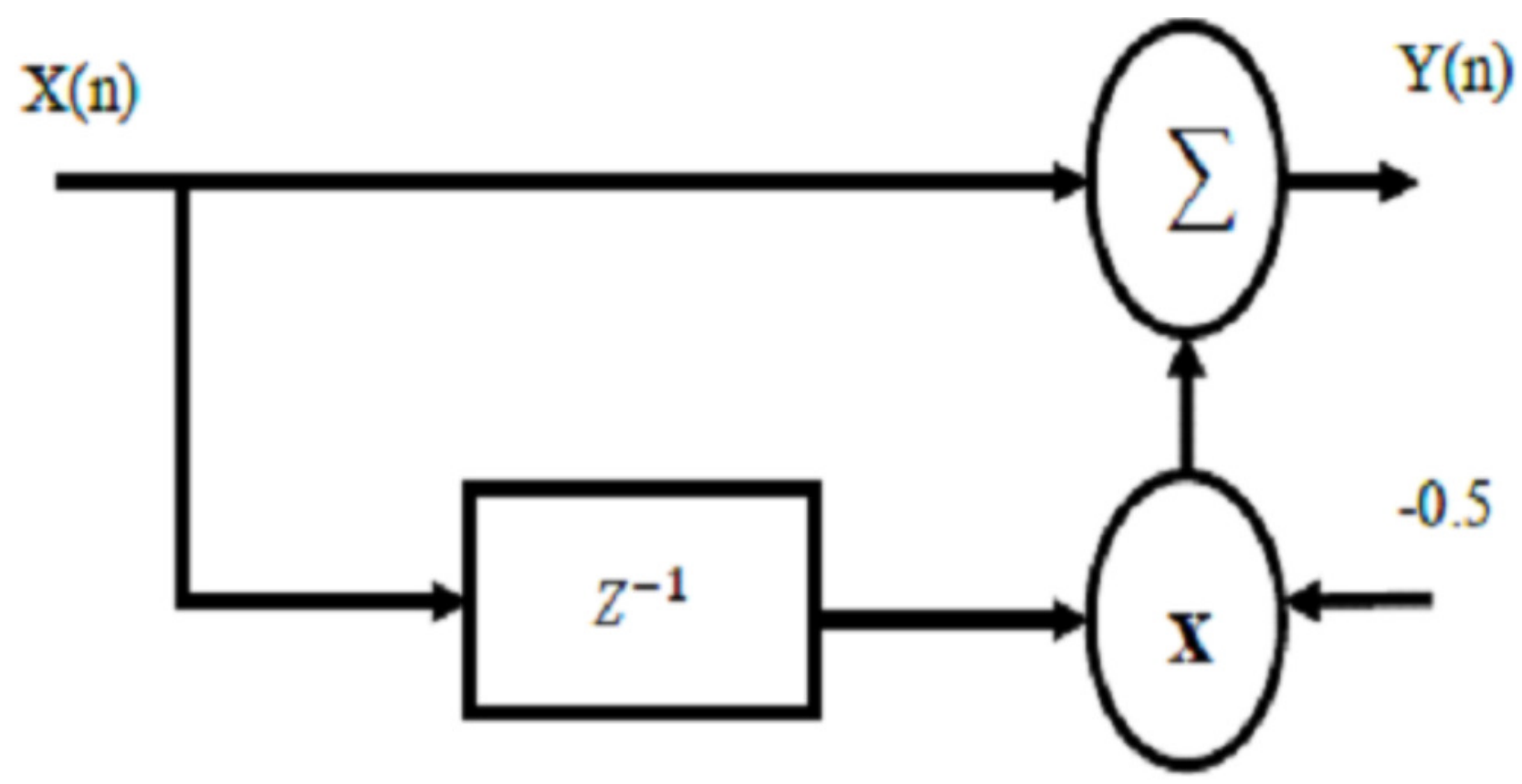


Figure 9

Block_diagram_of_the_proposed_processor_compared_with.

Block diagram of the proposed processor compared with the traditional 2D-FFT processor

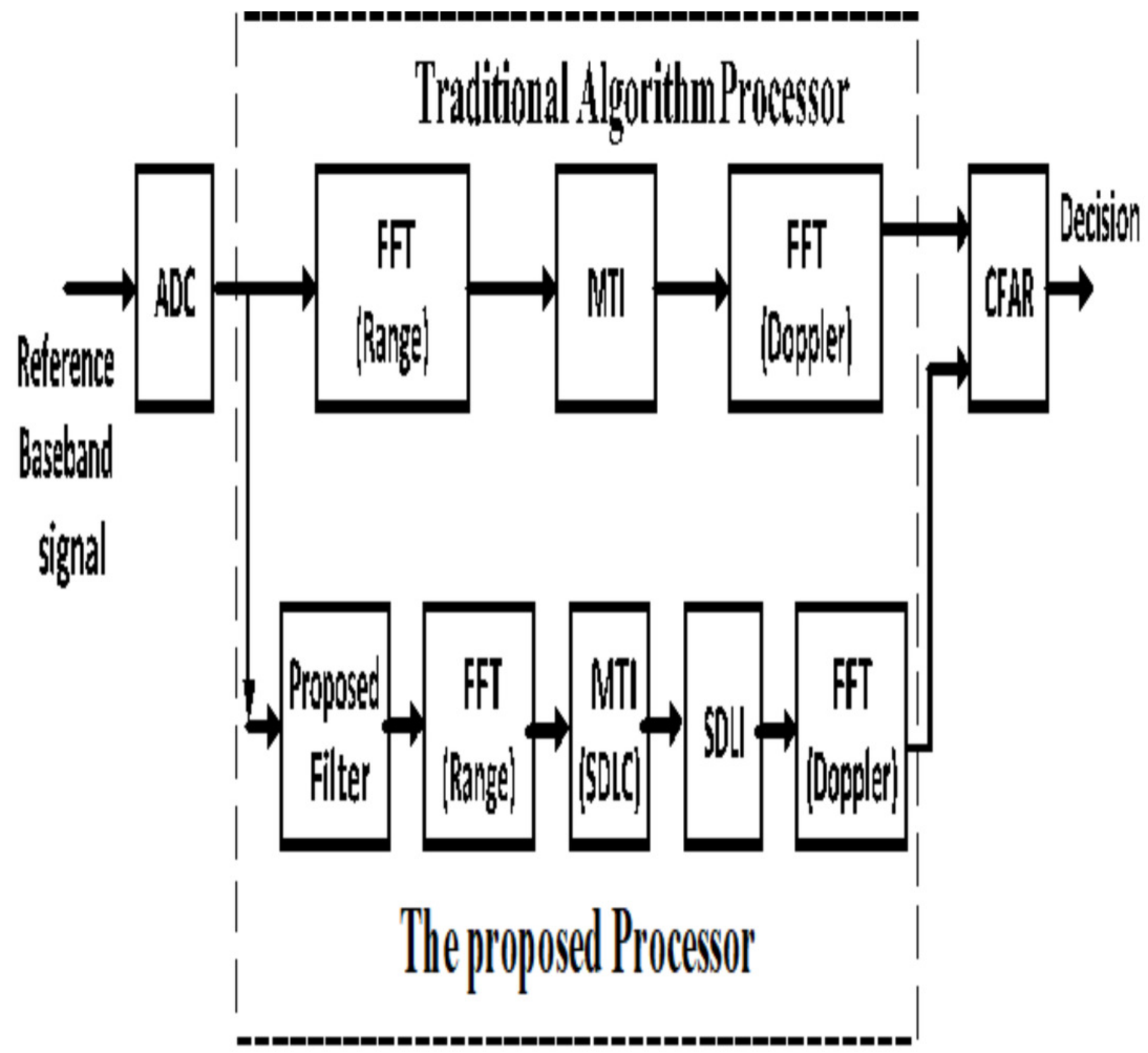




\section{Figure 10}

\section{Response_of_FFT_algorithm}

Response of FFT algorithm

(a) Before the proposed filter. (b) After the proposed filter. 


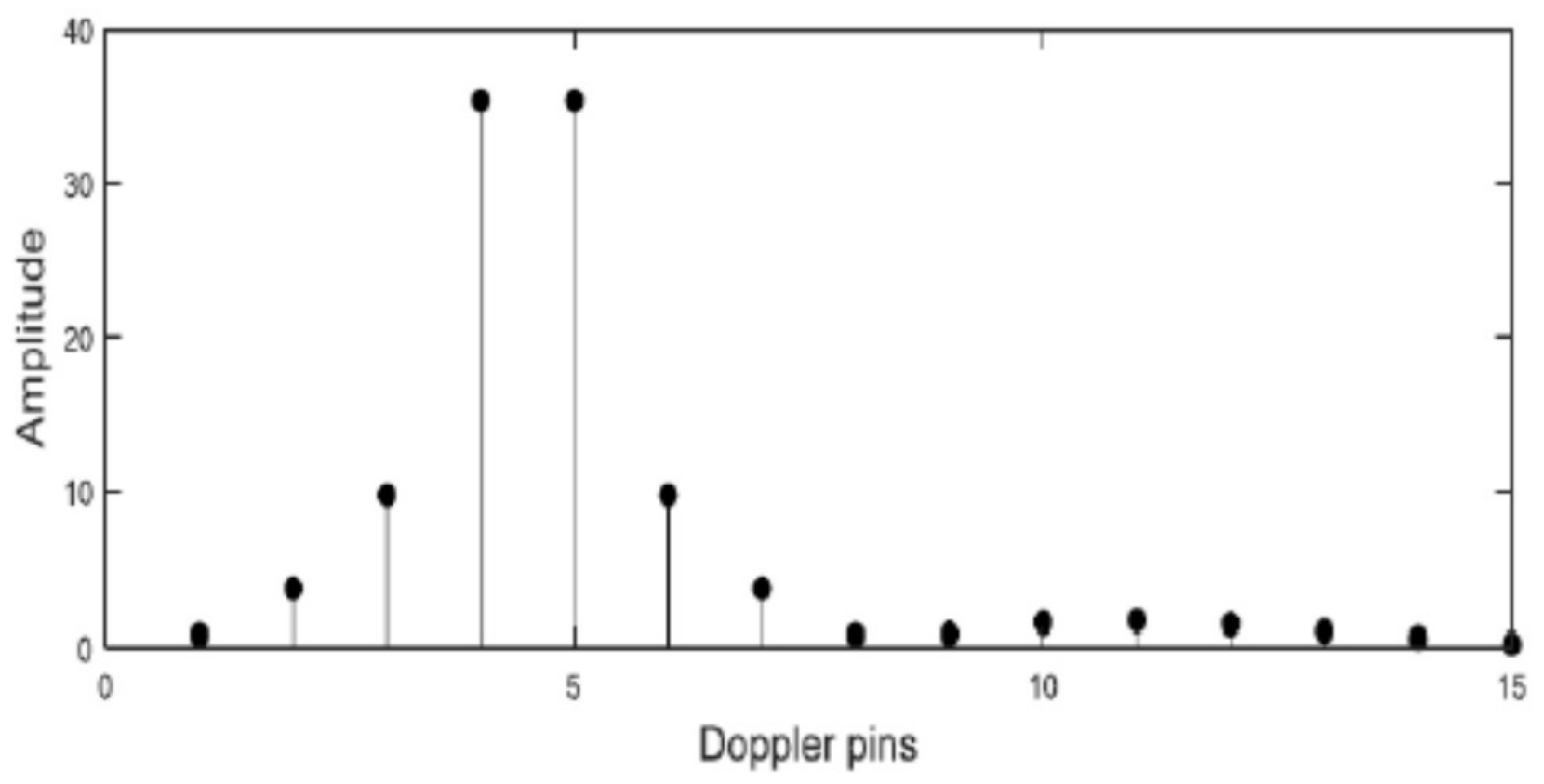

(a)

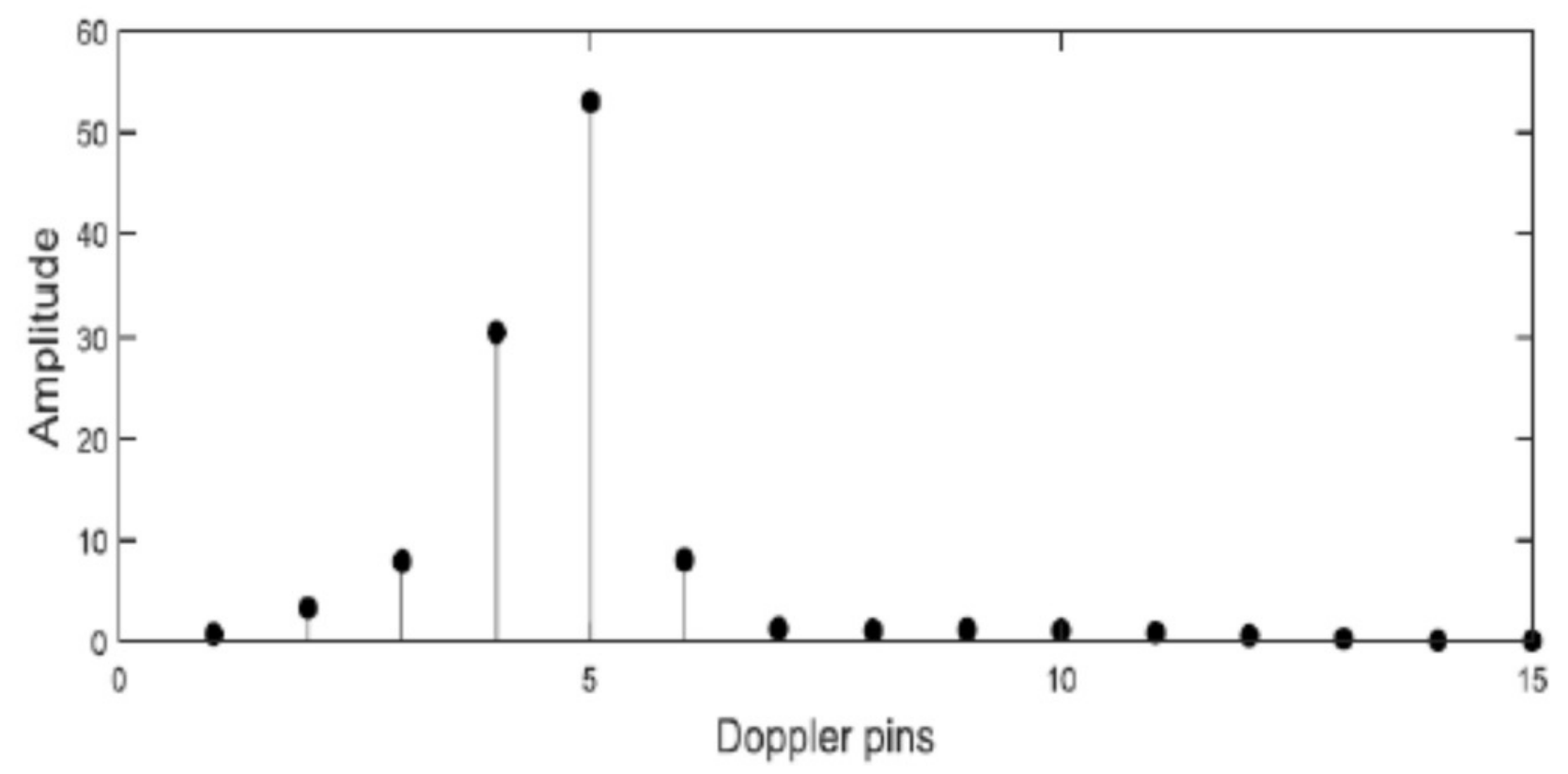

(b) 
Figure 11

Response_of_SDLC

Response of SDLC/SDLI processor compared with the traditional one at different Doppler frequencies

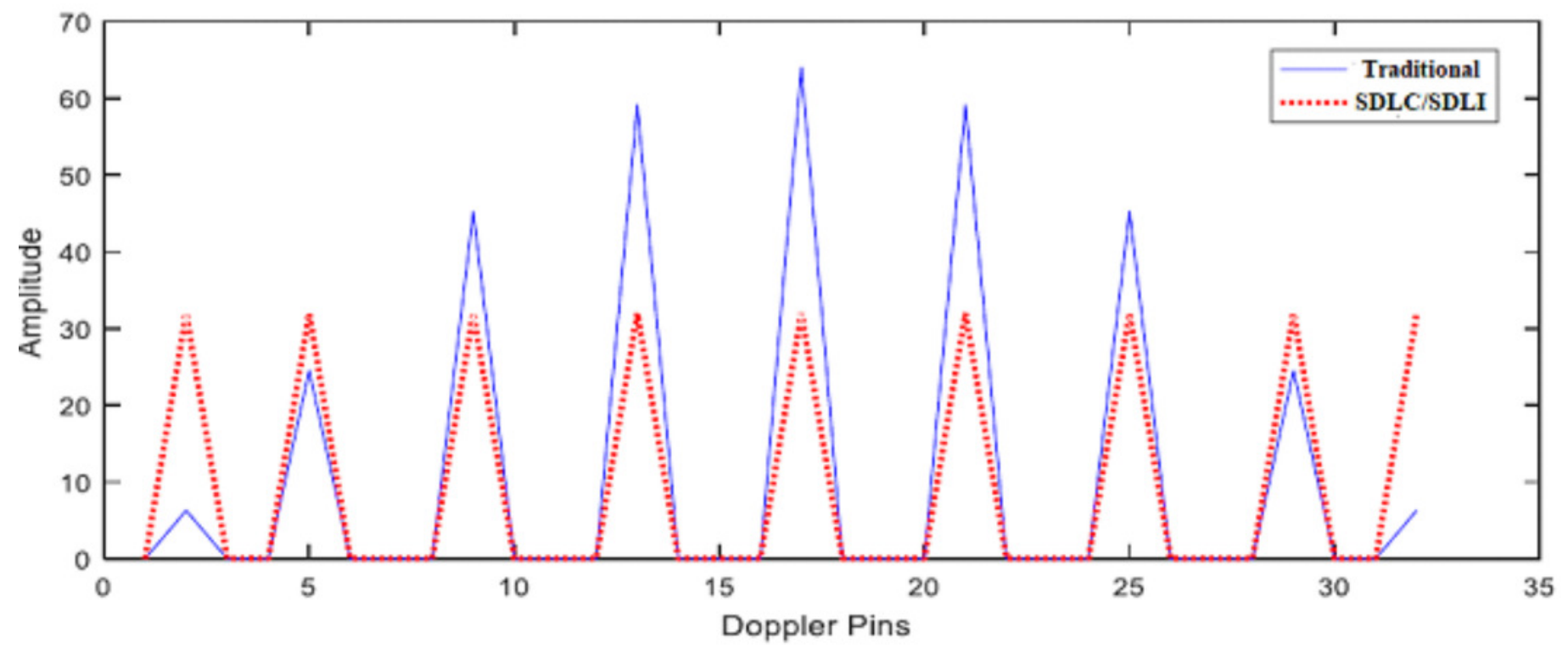


Figure 12

Response_of_the_proposed_processor

Response of the proposed processor compared with the traditional one at different Doppler frequencies

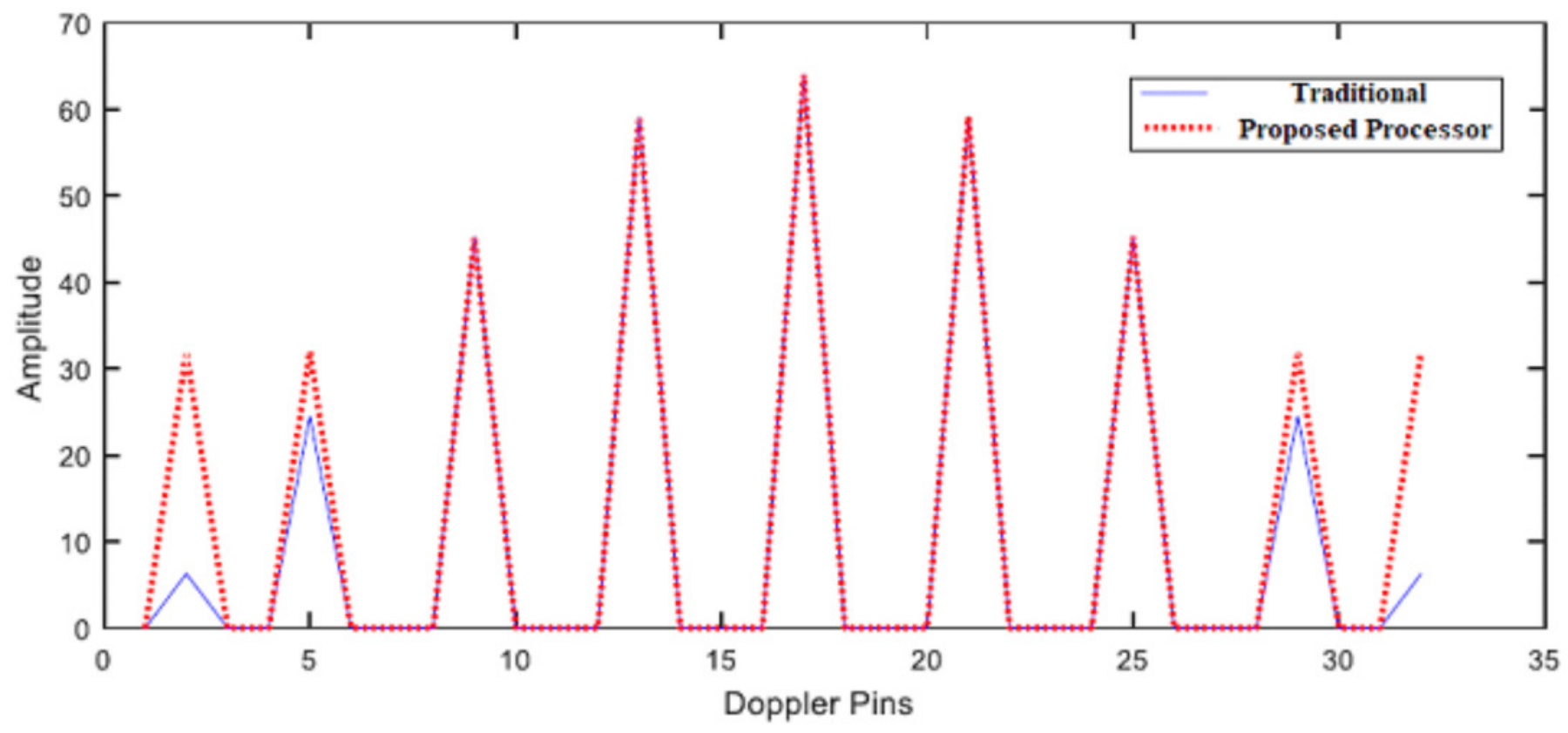


Figure 13

ROC_of_the_proposed_processor_for_slow_Doppler_target

ROC of the proposed processor compared with that of SDLC/SDLI and the traditional algorithms for slow Doppler target at Pfa of $10^{\wedge}-5$.

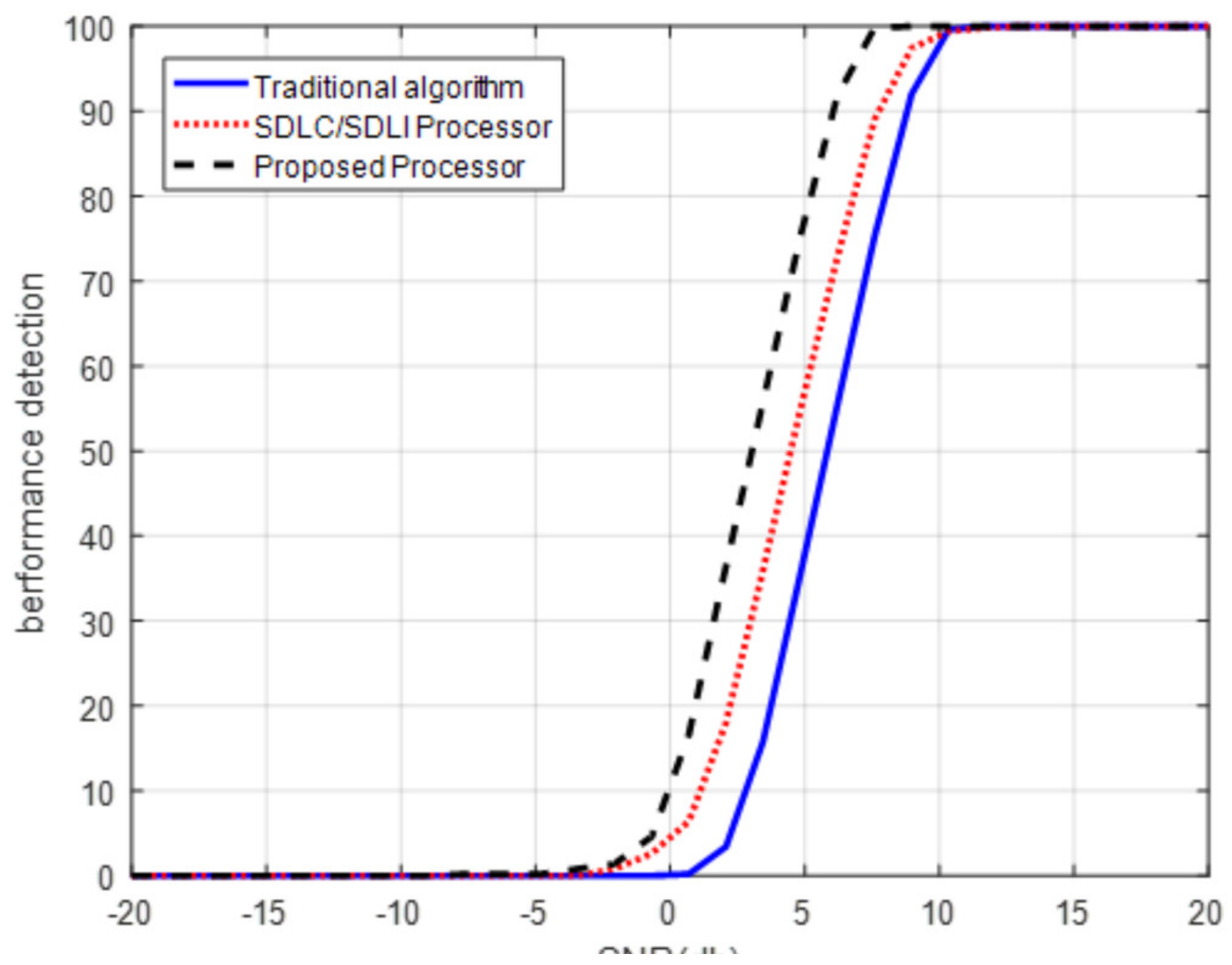


Figure 14

ROC_of_the_proposed_processor_for_middle_Doppler_target

ROC of the proposed processor compared with that of SDLC/SDLI and the traditional algorithms for middle Doppler target at Pfa of $10^{\wedge}-5$.

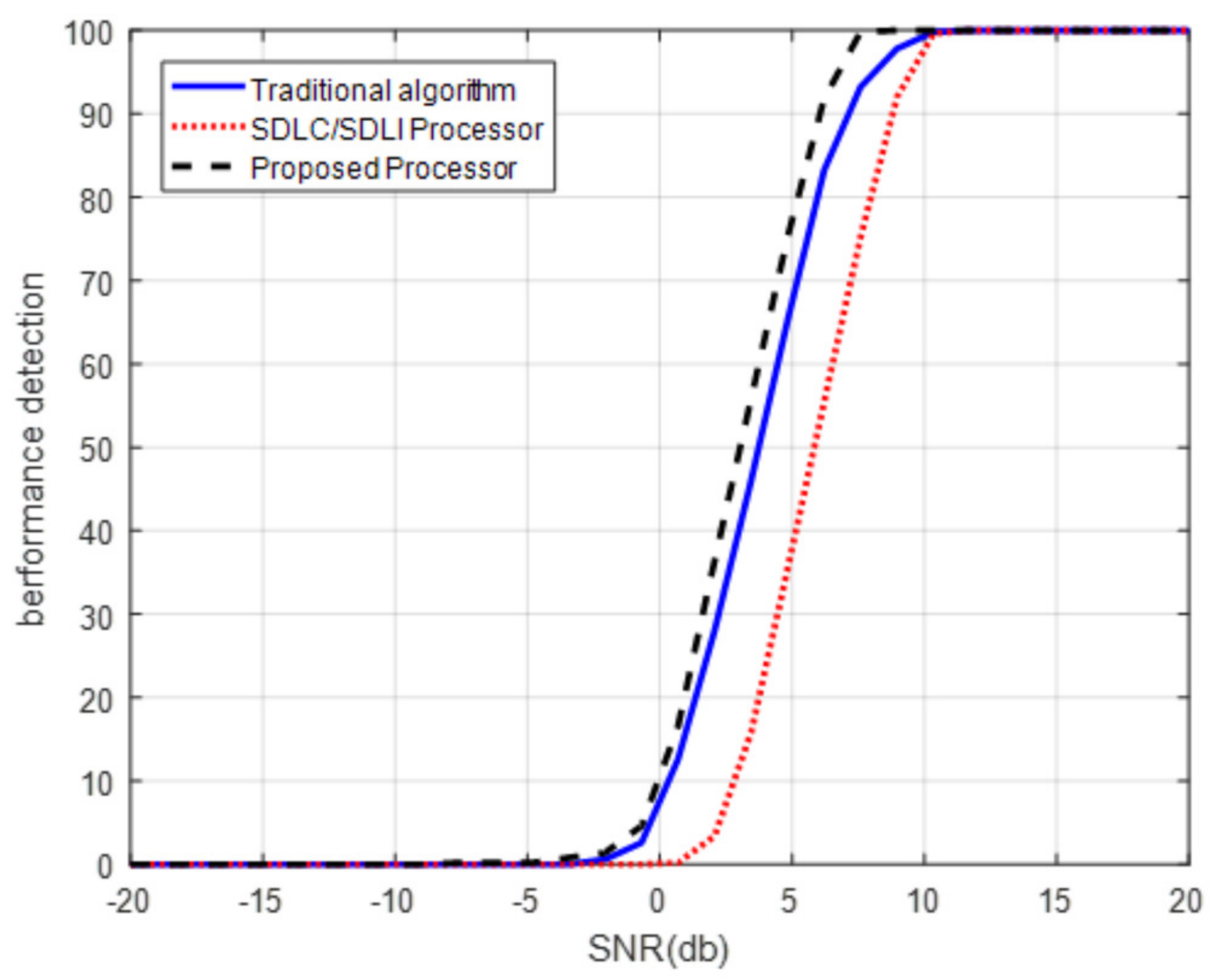




\section{Figure 15}

Simulation_results_of_target_detection_using_FPGA

Simulation results of target detection using FPGA

(a) Traditional algorithm

(b) Proposed processor

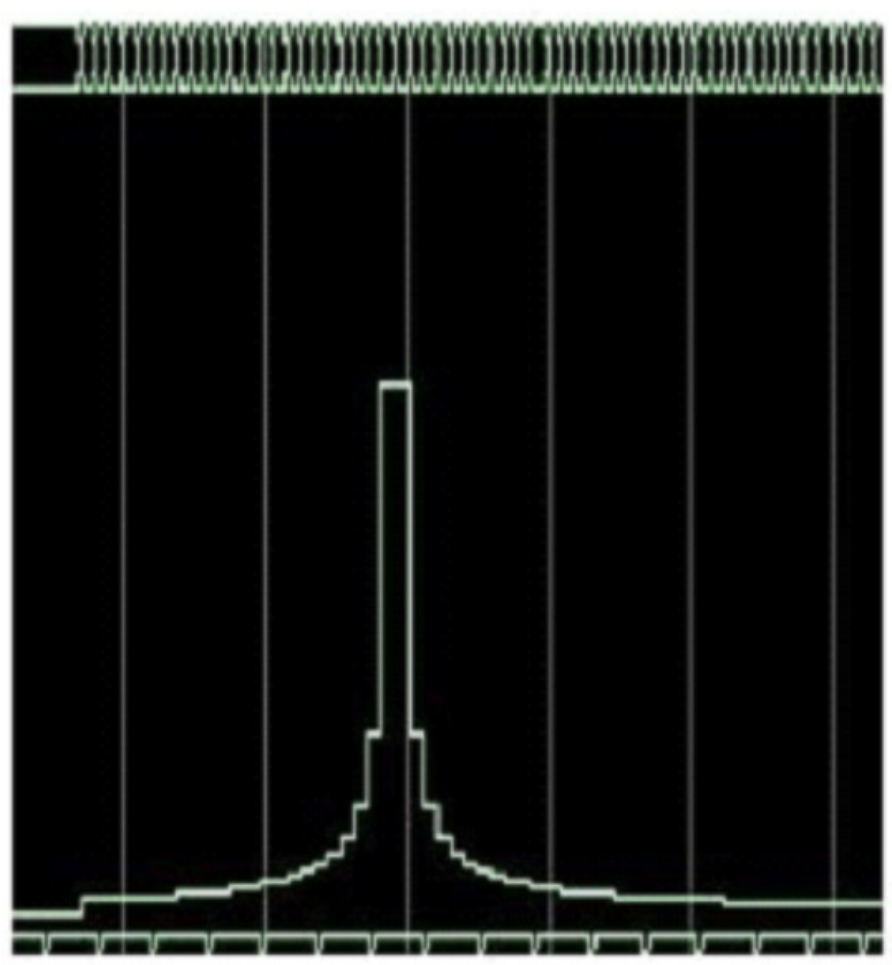

(a)

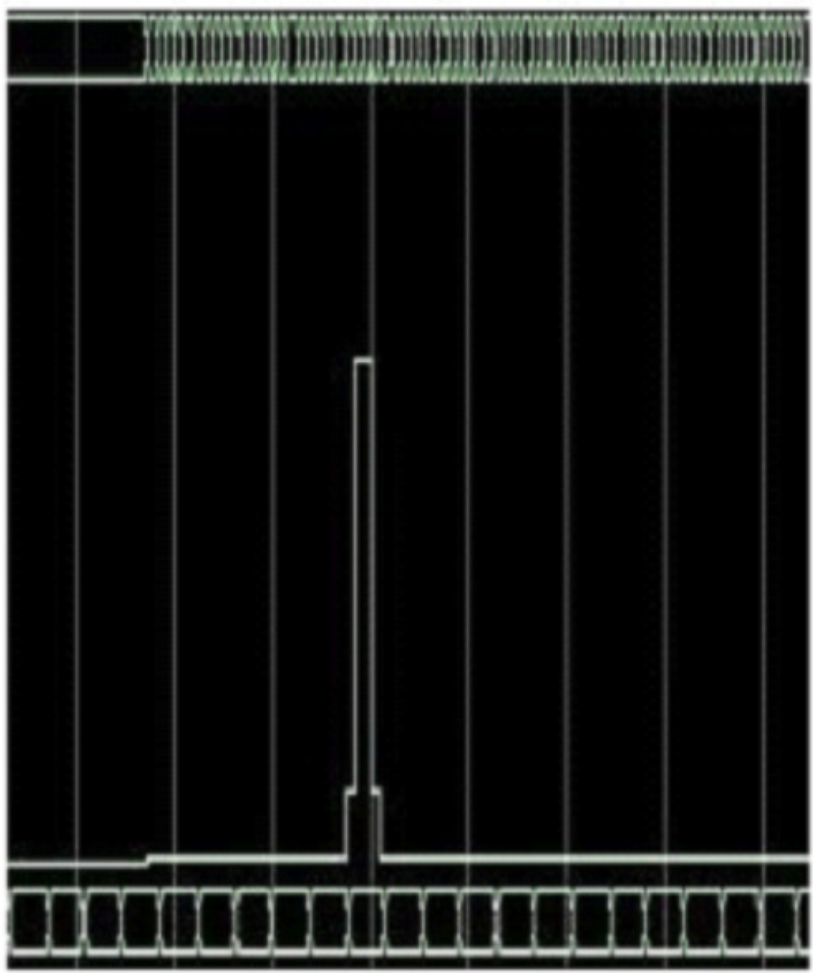

(b) 


\section{Figure 16}

Response of the proposed processor compared with that of both SDLC/SDLI

Response of the proposed processor compared with that of both SDLC/SDLI and traditional algorithms using FPGA

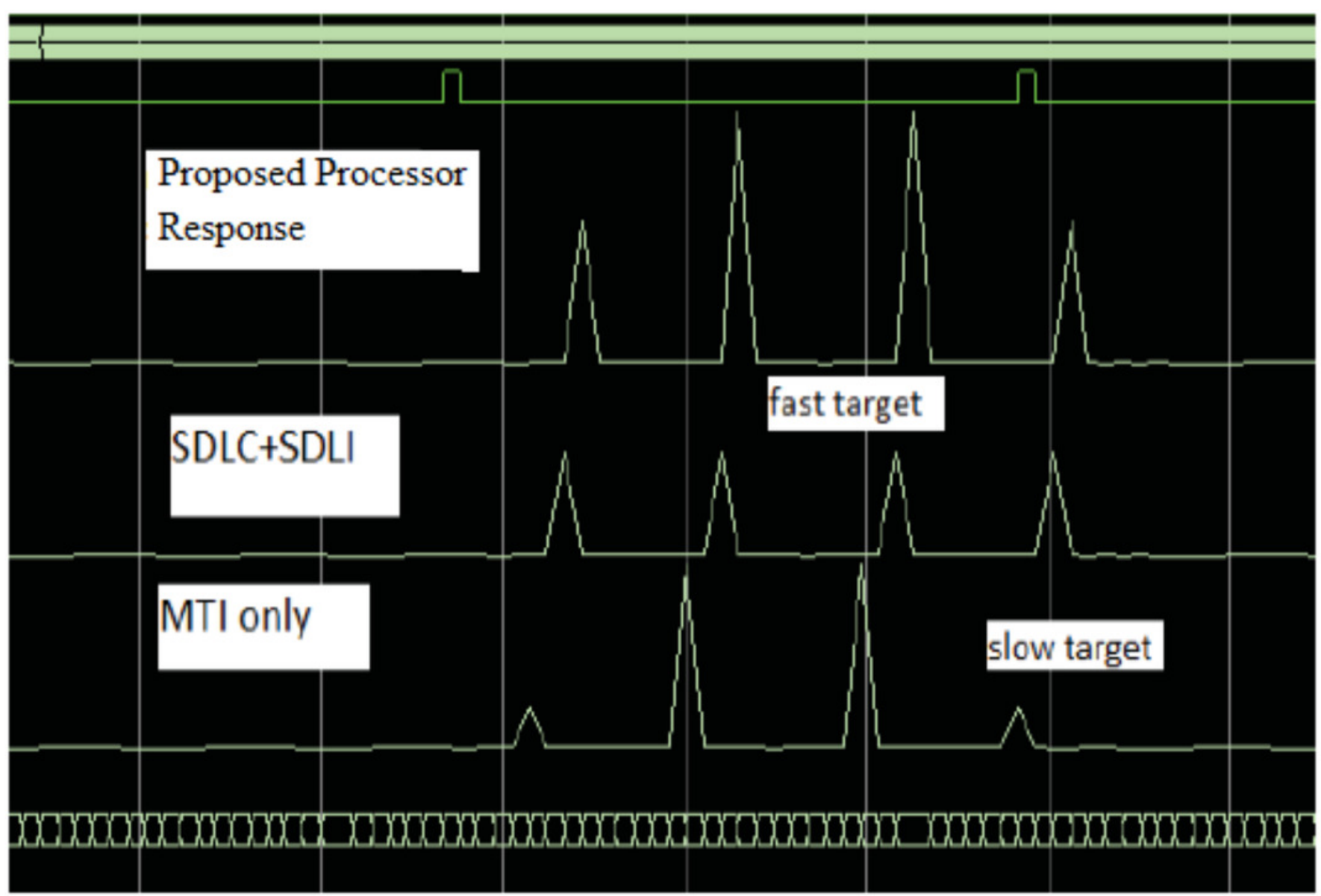


Figure 17

Chip scope result of the proposed processor

Chip scope result of the proposed processor, SDLC/SDLI and traditional responses.

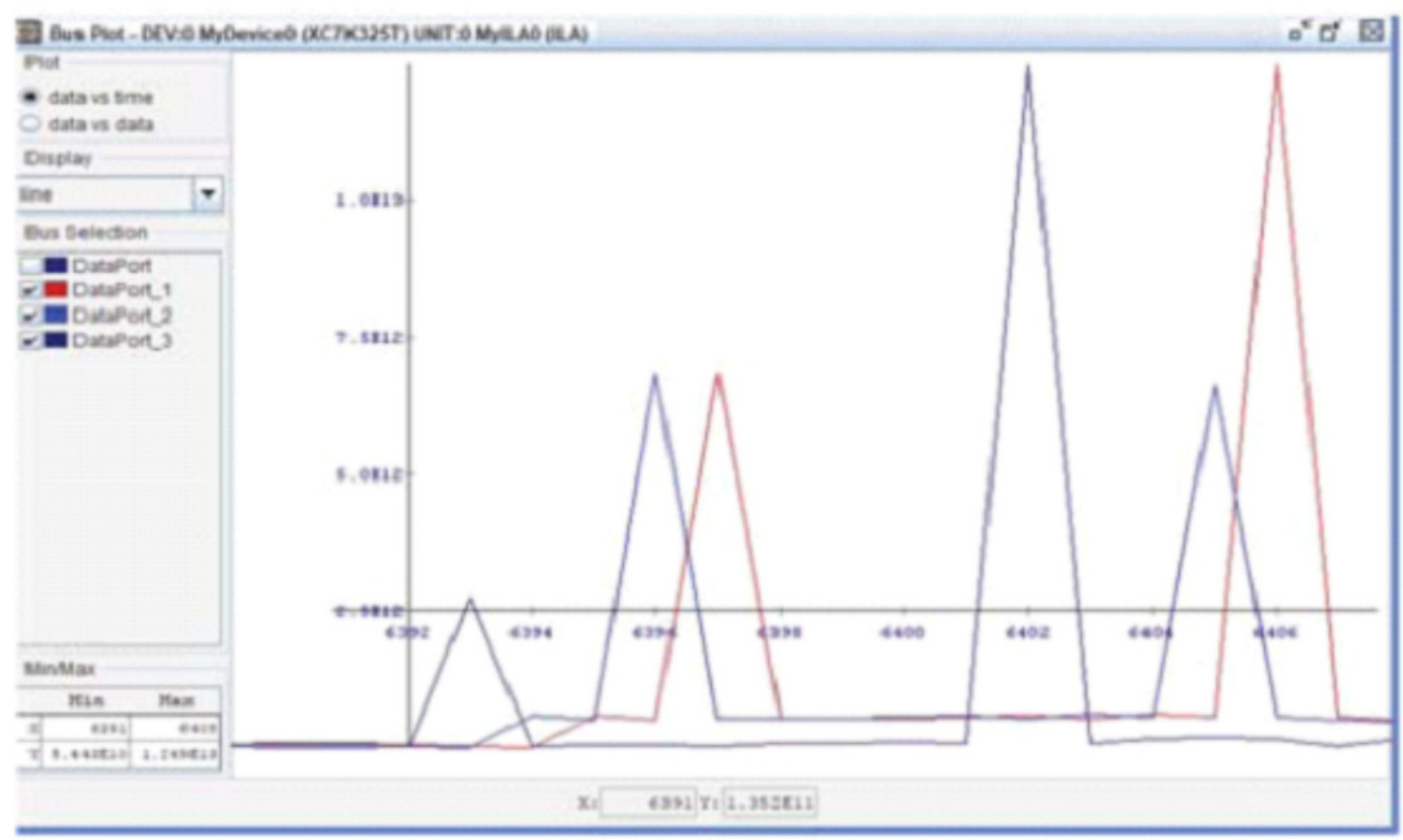

